2 Porter RA, Berrios GE. A History of Clinical Psychiatry: The Origin and History of Psychiatric Disease. Athlone Press, 1999.

Paul Wallang, Specialist Registrar in Forensic Psychiatry, John Howard Centre, East London NHS Foundation Trust, UK, email: paulwallang@hotmail.com

doi: $10.1192 / \mathrm{pb} .34 .7 .305 a$

\section{Too much reality television}

C. J. Jung, among others, commented that people cannot stand too much reality. Two recent admissions to our adult acute ward illustrate a related point regarding 'reality television'. Recent years have seen a marked increase in the quantity of such programmes, many of which contain content of a stigmatising nature.

The first case was a 24-year-old man who presented for the first time with persecutory delusions, including the belief that the hospital was a television studio. His family identified his appearance on a daytime talk show 3 months earlier as a contributory precipitant to this episode. During the programme the man had been exposed to a prolonged period of negative comments by the presenter in front of a live, as well as the TV, audience.

The second case was a 35-year-old man who had appeared on a talent show during which his audition performance had been severely criticised. He himself linked the subsequent deterioration in his self-esteem, and his feeling that people in his community saw him in a negative light, to the experience. His admission followed an episode of self-harm and he was admitted with predominantly depressive symptoms.

The British Medical Association has argued for the banning of boxing owing to the risks involved. I wonder whether the Royal College of Psychiatrists should take a similar view towards programmes that present public humiliation as entertainment.

Gary S. Hosty is a Consultant Psychiatrist at Shelton Hospital, Shrewsbury, email: hosty@tinyworld.co.uk

doi: $10.1192 / p b .34 .7 .306$

\section{Variable practice complicates standard setting for PICU prescribing}

Brown and colleagues ${ }^{1}$ rightly point out that there are minimal reference data against which psychiatric intensive care units (PICUs) can measure their own performance. We welcome their study, which describes the clinical activity of seven such units in England. What is particularly striking is their finding that there was a huge variability in prescribing practices between the units studied, which reached statistical significance in 14 of the 16 prescribing measures described. This included a tenfold variation in the rate of combination antipsychotic prescribing $(P<0.001)$ and a ninefold variation in the rate of high-dose antipsychotic prescribing $(P<0.005)$. The authors acknowledged that the rate of high-dose prescribing may have been underestimated owing to potential calculation errors.

As pointed out by Brown and colleagues, PICU patients are at a particularly high risk of neuroleptic malignant syndrome. Therefore, it is difficult to justify deviating from the evidence base for the particular conditions being treated, and practices such as combination prescribing and high-dose prescribing should be avoided if at all possible.

We question Brown et al's assertion that despite the wide variation in practice, and the potential calculation errors, their results are robust enough to serve as reference data for clinical governance purposes. Certainly, if these results are to be used as reference points, it needs to be clear which results should be used, i.e. the best results (e.g. $6 \%$ rate of combination prescribing) $v$. the combined percentages ( $23 \%$ rate of combination prescribing overall). Given the high variability between the units which participated in the study, perhaps other PICUs should be comparing themselves against the best results achieved, rather than the average.

A $6 \%$ rate of combination prescribing and a $2 \%$ (albeit an underestimate) rate of high-dose prescribing seem like standards that all PICUs should aspire to. Our experience is that such rates may well be achievable. We have achieved rates of $13 \%$ combination antipsychotic prescribing and $0 \%$ high-dose prescribing without any increase in our rate of violence (abstract in publication). We hope that the study performed by Brown and colleagues serves as a stimulus for further research and debate on the important issue of maintaining evidence-based practice, even when treating the most severely ill patients.

1 Brown S, Chhina N, Dye S. Use of psychotropic medication in seven English psychiatric intensive care units. Psychiatrist 2010; 34: 130-5.

Daniel P. Herlihy is ST5 in Psychiatry and Shubulade Smith is Consultant Psychiatrist working on a psychiatric intensive care unit in south London, South London and Maudsley NHS Foundation Trust, email: daniel.herlihy@slam.nhs.uk

doi: 10.1192/pb.34.7.306a

\title{
CORRECTION
}

The dois for several items in last month's correspondence column were printed incorrectly; the online versions have been corrected post-publication in deviation from print and in accordance with this correction.

Divine intervention in mental health (letter). The Psychiatrist 2010; 34: 258-259: doi: 10.1192/pb.34.6.258
Declarations of interest (letter). The Psychiatrist 2010; 34: 259: doi: 10.1192/pb.34.6.259

Declarations of interest (authors' reply). The Psychiatrist 2010; 34: 259: doi: 10.1192/pb.34.6.259a

BNF limits $v$. threshold dosing (letter). The Psychiatrist 2010; 34: 259-260: doi: 10.1192/pb.34.6.259b

doi: $10.1192 / p b .34 .7 .306 b$ 